\title{
Fairness guaranteed novel elCIC technology for capacity enhancement in multi-tier heterogeneous cellular networks
}

\author{
Qixun Zhang ${ }^{*}$, Tuo Yang, Yue Zhang and Zhiyong Feng
}

\begin{abstract}
Driven by surging demands for high data rate services and better user experiences, there is an increasing capacity demand in heterogeneous cellular networks. As one of the promising solutions for capacity enhancement, densely deployed small cells are proposed to provide a huge capacity gain and improve the user experience with high data rate services. However, the inter-cell interference among densely deployed cells is a big challenge that constraints the performance of capacity improvements in hierarchical multi-tier heterogeneous cellular networks. To minimize the inter-cell interference and achieve a fairness guaranteed solution among different users, a novel enhanced inter-cell interference coordination (elCIC) technology is proposed by jointly considering about the cell range expansion (CRE) scheme to minimize interferences among multi-tier cellular networks, improving the network throughput and quality of service (QoS). Optimal CRE bias and almost blank subframe (ABS) ratio solutions are achieved in this paper by considering the fairness among users at the center and cell edge. Moreover, the multi-objective decision-making problem is solved by maximizing the proportional fairness (PF) utility and area capacity in multi-tier heterogeneous cellular networks. Simulation results denote that a tradeoff between fairness and network throughput is achieved when CRE bias is from 8 to $12 \mathrm{~dB}$ and ABS ratio is from 4/8 to 6/8.
\end{abstract}

Keywords: Heterogeneous cellular networks; Small cell; CRE; elCIC; Multi-objective decision-making problem

\section{Introduction}

Recently, the rapid developments on various applications and different service demands in cellular networks lead to the exponentially surge on mobile traffics. According to statistics, the mobile traffic will grow up to 1,000 times $(1,000 \times)$ in the next 10 years. Moreover, the user data rate will also increase from approximately $1 \mathrm{Mbit} / \mathrm{s}$ today to at least $10 \mathrm{Mbit} / \mathrm{s}$ over the next decade in [1]. However, the explosion of mobile traffics brings in both new opportunities and challenges for the cellular network optimization for operators. In general, there are three ways to enhance the network capacity, such as the spatial densely deployment, spectral aggregation, and spectrum efficiency improvement. Spatial densely deployment is realized by deploying multi-tier overlayed heterogeneous

\footnotetext{
*Correspondence: zhangqixun@bupt.edu.cn Key Lab. of Universal Wireless Communications Ministry of Education, Information and Telecommunication Engineering of Beijing University of Posts and Telecommunications (BUPT), Haidian Dist. Xitucheng Rd., Beijing 100876 , P.R. China
}

networks (HetNets) to improve the network capacity and ensure the cell coverage in [2]. Spectral aggregation refers to the utilization of carrier aggregation (CA) technology to support a wide system bandwidth up to $100 \mathrm{MHz}$ in [3]. In terms of the spectrum efficiency, it can be improved by introducing new transmission technologies such as cooperative multipoint (CoMP) in [4]. Among these technologies, HetNet is the most effective and low-cost solution to improve the capacity in $[5,6]$ by deploying small cells within multi-tier heterogeneous cellular networks.

In the multi-tier HetNet scenario, low-power small cells, such as femto, pico, and relay nodes, are overlaid within the coverage of macrocells, which are placed in an unplanned manner in [7]. Compared with macrocell, small cells have much lower transmit power, smaller coverage, and lower cost which are easy to maintain and deploy. Small cells can alleviate the traffic pressure of macrocell network by offloading part of the traffic to them. However, HetNet will face a series of problems as well, which will affect the capacity enhancement of

\section{是 Springer}

(c) 2015 Zhang et al.; licensee Springer. This is an Open Access article distributed under the terms of the Creative Commons Attribution License (http://creativecommons.org/licenses/by/4.0), which permits unrestricted use, distribution, and reproduction in any medium, provided the original work is properly credited. 
multi-tier heterogeneous cellular networks. In [8], some cooperative distributed radio resource management algorithms for time synchronization, carrier selection, and power control were discussed for hyper-dense small cell deployment. Among these challenges of hyper-dense HetNet, traffic imbalance and inter-cell interference among multiple cells are two tough problems which lead to the inefficient radio resource utilization and affect the capacity and sum data rate of HetNet in [9].

To solve these problems, both cell range expansion (CRE) and enhanced inter-cell interference coordination (eICIC) technologies were proposed to improve the network capacity of HetNet in the 3GPP Release 8 and Release 9. If adopt the maximum reference signal received power (RSRP) access scheme, only a few users can be associated with low power nodes, which will cause the load imbalance among cells in HetNets and greatly reduce the capacity gain from small cell deployment. Therefore, a positive bias value is added to the RSRP of user equipments (UE) by using the CRE technology in [10], which can offload part of macrocell UE (MUE) to small cells and reduce the uplink interference among multi-tier networks. However, small cells serving the range expanded UEs with a low value of signal to interference plus noise ratio (SINR) will surfer serious downlink interference from macrocells, which is a critical problem unsolved yet.

In terms of the eICIC technology, it can efficiently improve the range expanded UEs' performance and reduce the inter-cell interference among cells in multitier heterogeneous cellular networks. As a time-domain interference coordination scheme, the eICIC technology divides the time frames into almost blank subframes (ABS) and normal subframes in [11]. And MUEs and range expanded UEs are scheduled on normal subframes and $\mathrm{ABSs}$ orthogonally, which can relieve the interference from macrocells to the range expanded UEs and improve the quality of service (QoS). But the eICIC technology reduces the time resources of MUEs for communication at the same time.

In the literature, the joint usage of CRE and eICIC is proved to be an effective solution on improving the system throughput of HetNet in $[12,13]$, but it is much more complicated for mathematical analysis of network capacity. Besides, if a larger CRE bias of RSRP is applied to reduce the load imbalance among different cells by the traffic offloading technology, more ABSs are needed in order to guarantee the performance of range expanded UEs. Therefore, the CRE bias and ABS muting ratio are key parameters that should be configured appropriately to ensure the performance on the interference mitigation and the network capacity enhancement using the joint CRE and eICIC schemes. In [14], a joint optimization of the CRE bias and radio resources is discussed to ensure the performance of picocells. However, the over-the-air signaling has not been considered yet which leads to the numerical optimization, and investigation results are unpractical. Moreover, the performance of CRE and eICIC schemes is studied and evaluated by system simulation results in [15], which lacks the mathematical analysis and does not consider about the configuration of CRE and eICIC. Furthermore, the analytical approaches for CRE biasing and eICIC are studied in [16-20]. The SINR of HetNet with cell association is studied in [16], but it does not include the resource partitioning scheme and only analyzes the SINR. And the SINR and mean throughput-based analysis for resource partitioning are analyzed in $[17,18]$, without considering the user association effect. Optimal CRE bias and ABS ratio scheme are proposed in [19] based on the average per user spectral efficiency. In [20], the joint analysis of resource partitioning and offloading scheme is proposed in terms of the metrics of the effective distribution of SINR but has no consideration about the optimal parameter configuration effects on UE's fairness. Considering fairness, a graph-based distributed algorithm called fairness guaranteed cooperative resource allocation (FGCRA) is proposed to manage the interference among femtocells in [21], but it only considers the sub-channel allocation which has not considered about the time resource.

Considering the disadvantages of existing works and problems unsolved, a novel eICIC technology is proposed by jointly considering the CRE scheme to minimize interferences among multi-tier cellular networks in this paper, improving the network throughput and QoS and guarantee the fairness of users meanwhile. Both the poisson point process (PPP) model and two metrics of capacity performance are used including the average capacity per cell and the average capacity per area unit. Optimal CRE bias and ABS ratio are achieved by taking into account the fairness effect of users at the center and cell edge. Furthermore, the multi-objective decisionmaking problem is solved to maximize the proportional fairness (PF) utility [22] and area capacity of multi-tier heterogeneous cellular networks. Simulations are performed to evaluate the performance of proposed technology in this paper. The tradeoff between fairness and network throughput is achieved in this paper, when the CRE bias is from 8 to $12 \mathrm{~dB}$ and $\mathrm{ABS}$ ratio is from $4 / 8$ to $6 / 8$.

The remainder of this paper is organized as follows. Both the system model and problem formulation of heterogeneous cellular networks are proposed in Section 2. The optimal solution of joint CRE bias and ABS ratio technology is proposed in Section 3 by considering about the fairness effect among UEs. Results are discussed and analyzed thoroughly in Section 4. Finally, a brief conclusion is described in Section 5. 


\section{System model and problem formulation}

A typical scenario of one macrocell overlapped by small cells, such as the picocells, is described in detail in this section. Besides, the problem formulations of downlink capacity and interference are proposed by taking into account the macrocells and small cells in multi-tier heterogeneous cellular networks.

\subsection{System model and scenario}

The typical scenario of multi-tier heterogeneous cellular networks is denoted in Figure 1, where picocells are deployed randomly within the coverage of macrocell. To achieve the goal of load balance, CRE technology is proposed to improve the system capacity. However, UEs at the edge of picocell will suffer the strong co-channel interference in downlink from the macrocell. Therefore, in order to minimize the co-channel interference among picocell and macrocell, the eICIC technology is utilized to enhance the performance of picocell UE (PUE) by jointly using CRE technology at the cell edge as denoted by PUE $_{\text {cre }}$.

As a time-domain interference coordination mechanism, subframes are divided into ABS and normal subframes. Macrocell mutes its data transmission on specific subframes, where picocell schedules $\mathrm{PUE}_{\text {cre }}$ on the corresponding subframes to avoid co-channel interferences from macrocell as shown in Figure 2.

\subsection{Problem formulation}

In multi-tier heterogeneous cellular networks, the positions of base stations (BSs) are denoted by $\Phi_{k}$ in the $k$ th tier which follows a homogeneous poisson point process (PPP) distribution with a intensity of $\lambda_{k}$. In our discussion, the first tier $(k=1)$ represents macrocells and the second tier $(k=2)$ represents picocells. Moreover, the positions of UEs are depicted by $\Phi_{u}$ which follows a homogeneous PPP distribution with a intensity of $\lambda_{u}$ and is independent of $\Phi_{k}$. The transmit power of each BS is assumed as the same and denoted by $P_{k}$ with the bandwidth of $W$. And

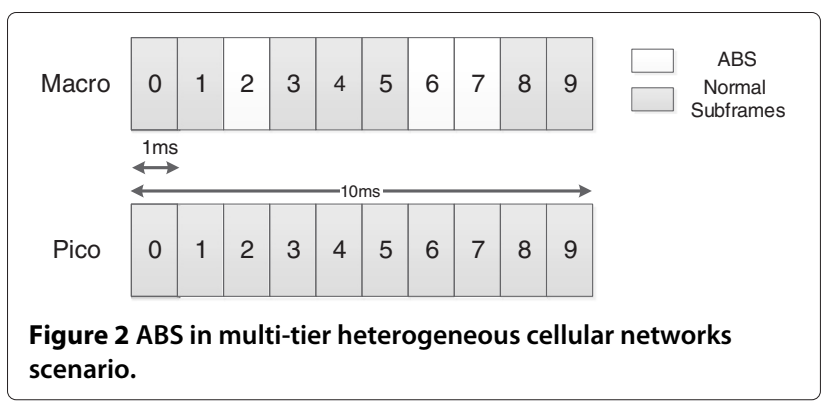

the path loss ratio is $\left\{\alpha_{k}\right\}=4$. Assumed that the multi-tier heterogeneous cellular network is an interference limited system, the inter-cell interference is dominant and the background noise is ignored for analysis simplicity in this paper.

\subsubsection{Cell association using CRE technology}

In order to offload the high volume traffics from macrocell, CRE technology with different CRE bias values is utilized in macrocell and picocell with $B_{1}=0 \mathrm{~dB}$ and $B_{2}=B, B>0 \mathrm{~dB}$. Considering the cell association, different users will choose the appropriate serving BS in the $k$ th tier with the strongest RSRP value as $P_{r, k}=P_{k} h Z_{k}{ }^{-\alpha_{k}} B_{k}$, where $Z_{k}$ denotes the distance between UE and BS and $h$ is the channel gain with a Rayleigh distribution, i.e., $h \sim \exp (1)$. Therefore, both the transmit power ratio of BS and the CRE bias ratio of interfering BS to serving BS are shown in Equation 1.

$$
\hat{P}_{j}=\frac{P_{j}}{P_{k}}, \hat{B}_{j}=\frac{B_{j}}{B_{k}} .
$$

In terms of different CRE bias values, UEs are divided into three types.

Type $1 U_{1}$ : MUE associated to $k=1$ tier. The RSRP is correspond to $P_{r, 1}>B P_{r, 2}$.

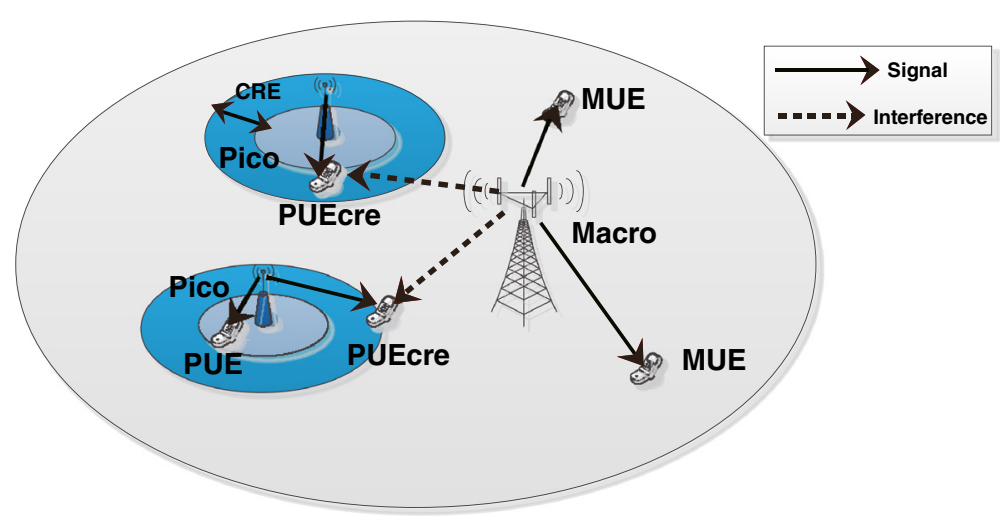

Figure 1 Scenario of multi-tier heterogeneous cellular networks. 
Type $2 U_{2}$ : PUE associated to $k=2$ tier at the center of picocell without CRE. The RSRP is correspond to $P_{r, 1}<$ $P_{r, 2}$.

Type $3 U_{\text {cre }}$ : Picocell UE using CRE (PUE cre $)$ associated to $k=2$ tier at the cell edge of picocell with CRE. The RSRP is correspond to $P_{r, 2}<P_{r, 1}<B P_{r, 2}$.

When the ABS configuration ratio is $\beta$ in macrocell, the time resource ratio of MUE is denoted by $\rho_{1}=1-\beta$, and the time resource ratio values of PUEs at the center and cell edge are denoted by $\rho_{2}=1$ and $\rho_{\text {cre }}=\beta$. Moreover, the UEs' proportion of three types is depicted by $A_{k}$ in Equation 2 , and $A_{1}, A_{2}$, and $A_{\text {cre }}$ corresponding to $U_{1}, U_{2}$, and $U_{\text {cre }}$ are denoted in Equations 3 to 5 , which is affected by key parameters including the transmit power, density, and CRE bias values. Furthermore, the UEs' average number of three types is $N_{k}=\frac{\lambda_{u} A_{k}}{\lambda_{k}}$ as denoted in Equations 6 to 8 .

$$
\begin{aligned}
& A_{k} \triangleq \mathrm{P}\left(\bigcup_{k=1,2 \cdots, K j \neq k} P_{r, k}>P_{r, j}\right) \\
& A_{1}=\frac{\lambda_{1} \sqrt{P_{1}}}{\lambda_{1} \sqrt{P_{1}}+\lambda_{2} \sqrt{P_{2} B}} \\
& A_{2}=\frac{\lambda_{2} \sqrt{P_{2}}}{\lambda_{1} \sqrt{P_{1}}+\lambda_{2} \sqrt{P_{2}}} \\
& A_{\text {cre }}=\frac{\lambda_{2} \sqrt{P_{2} B}}{\lambda_{1} \sqrt{P_{1}}+\lambda_{2} \sqrt{P_{2} B}}-\frac{\lambda_{2} \sqrt{P_{2}}}{\lambda_{1} \sqrt{P_{1}}+\lambda_{2} \sqrt{P_{2}}} \\
& N_{1}=\frac{\lambda_{u} \sqrt{P_{1}}}{\lambda_{1} \sqrt{P_{1}}+\lambda_{2} \sqrt{P_{2} B}} \\
& N_{2}=\frac{\lambda_{u} \sqrt{P_{2}}}{\lambda_{1} \sqrt{P_{1}}+\lambda_{2} \sqrt{P_{2}}} \\
& N_{\text {cre }}=\frac{\lambda_{u} \sqrt{P_{2} B}}{\lambda_{1} \sqrt{P_{1}}+\lambda_{2} \sqrt{P_{2} B}}-\frac{\lambda_{u} \sqrt{P_{2}}}{\lambda_{1} \sqrt{P_{1}}+\lambda_{2} \sqrt{P_{2}}} .
\end{aligned}
$$

\subsubsection{Resource partitioning using eICIC technology}

To minimize the interference from macrocell to PUEs, the eICIC technology is utilized which is a time resource partitioning approach. The macrocell mutes its transmission on certain fraction of subframes, picocell schedule UEs in the range expanded regions on the corresponding subframes to avoid the interference from the macrocell. Furthermore, macrocell configures its ABS with the ratio $\beta$. Thus, the time resource ratio of MUE is $\rho_{1}=1-\beta$, the time resource ratio of $\mathrm{PUE}_{\text {cre }}$ is denoted by $\rho_{\text {cre }}=\beta$, and the time resource ratio of PUE that is not affected by ABS is denoted by $\rho_{2}=1$.

Considering the interference that $\mathrm{PUE}_{\text {cre }}$ suffers form macrocells and other picocells without using the eICIC technology, the SINR of PUE cre $_{\text {is depicted in Equation } 9}$ where $\mathrm{BS}_{k 0}$ is $\mathrm{PUE}_{\mathrm{cre}}$ 's serving BS. In contrast, by utilizing the eICIC technology, the SINR of PUE cre $_{\text {only suffer }}$ the interference from other picocells, which is depicted in Equation 10. Therefore, the eICIC technology is an effective solution to reduce the interference from macrocell to $\mathrm{PUE}_{\text {cre }}$ in multi-tier heterogeneous cellular networks.

$$
\begin{aligned}
& \operatorname{SINR}(x)=\frac{P_{2} h_{2} x^{-\alpha}}{\sum_{j=1}^{2} \sum_{i \in \phi_{j} \backslash \mathrm{BS}} P_{k 0} h_{j} x^{-\alpha}} \\
& \operatorname{SINR}(x)=\frac{P_{2} h_{2} x^{-\alpha}}{\sum_{i \in \phi_{2} \backslash \mathrm{BS}_{k 0}} P_{2} h_{2} x^{-\alpha}}
\end{aligned}
$$

Furthermore, $X_{k}$ denotes the distance between UE and its serving BS in the $k$ th tier in multi-tier heterogeneous cellular networks, and the probability of $X_{k}>x$ is denoted in Equation 11. And BSs are deployed with a homogeneous PPP distribution model, where $X_{k}$ is a random variable with the probability density function in Equations 12 to 14 of $U_{1}, U_{2}$, and $U_{\text {cre }}$.

$$
P\left(X_{k}>x\right)=P\left(Z_{k}>x \mid \text { associated to } k \text { th tier }\right)=\frac{P\left(Z_{k}>x\right)}{A_{k}}
$$

$$
\begin{aligned}
f_{X_{1}}(x)=\frac{2 \pi \lambda_{1}}{A_{1}} x \exp \left[-\pi\left(\lambda_{1}+\lambda_{2} \sqrt{\frac{P_{1}}{P_{2} B}}\right) x^{2}\right] \\
f_{X_{2}}(x)=\frac{2 \pi \lambda_{2}}{A_{2}} x \exp \left[-\pi\left(\lambda_{2}+\lambda_{1} \sqrt{\frac{P_{1}}{P_{2}}}\right) x^{2}\right] \\
f_{X_{\text {cre }}}(x)=\frac{2 \pi \lambda_{2}}{A_{\text {cre }}} x\left\{\exp \left[-\pi\left(\lambda_{2}+\lambda_{1} \sqrt{\frac{P_{1}}{P_{2} B}}\right) x^{2}\right]\right. \\
\left.-\exp \left[-\pi\left(\lambda_{2}+\lambda_{1} \sqrt{\frac{P_{1}}{P_{2}}}\right) x^{2}\right]\right\}
\end{aligned}
$$

\subsection{Capacity analysis using CRE and eICIC technologies}

Considering the interference problem of heterogeneous cellular networks, a novel technology by jointly using CRE and eICIC schemes are proposed to improve the system capacity by minimizing the inter-cell interferences. Therefore, the downlink capacity per area unit of using the joint CRE and eICIC scheme is affected by different key parameters, such as the BS density $\lambda_{k}$, CRE bias $B$, and time resource $\rho_{k}$. And the average capacity per area unit is depicted by $C_{a}$ in Equations 15. $\rho_{k}$ is the time resource ratio, $\lambda_{k}$ is the BS density of $k$ th tier, $R_{k}$ is the average ergodic rate in [16], and $W_{k}$ is the bandwidth of $k$ th tier.

$$
C_{a}=\sum_{k=1}^{K} \rho_{k} \lambda_{k} R_{k} W_{k}
$$


Moreover, the average ergodic rate of UE of the $k$ th type is depicted by $R_{k}$ in Equation 16 , where $x$ is the distance between UE and BS. By applying the CRE scheme, $R_{k}$ is denoted in Equations 17 to 18 according to [16], where $C_{k}=\sum_{j=1}^{2} \lambda_{j} \sqrt{\hat{P}_{j} \hat{B}_{j}}, C_{\mathrm{cre} 1}=\lambda_{2}+$ $\lambda_{1} \sqrt{P_{1} / P_{2} B}, C_{\text {cre2 }}=\lambda_{2}+\lambda_{1} \sqrt{P_{1} / P_{2}}, C(t)=\sum_{j=1}^{2}$ $\lambda_{j} \sqrt{\left(e^{t}-1\right) \hat{P}_{j}} \arctan \left(\sqrt{e^{t}-1 / \hat{B}_{j}}\right), C(t)^{\prime}=\lambda_{2} \sqrt{\left(e^{t}-1\right)}$ $\arctan \left(\sqrt{e^{t}-1}\right)$.

$$
\begin{aligned}
& R_{k} \triangleq E_{x}\left\{E_{\mathrm{SINR}_{k}}\left[\ln \left(1+\operatorname{SINR}_{k}(x)\right)\right]\right\} \\
& R_{k}=\frac{\lambda_{k}}{A_{k}} \int_{0}^{\infty} \frac{1}{C_{k}+C(t)} d t \quad k=1,2 \\
& R_{\text {cre }}=\frac{\lambda_{2}}{A_{\text {cre }}} \int_{0}^{\infty} \frac{1}{C_{\text {cre } 1}+C(t)}-\frac{1}{C_{\text {cre } 2}+C(t)} d t
\end{aligned}
$$

Furthermore, the eICIC scheme using ABS configuration is utilized in order to decrease inter-cell interference from macrocell BS (MBS) to PUEs. Thus, the average ergodic rate is denoted by $R_{1}{ }^{\prime}, R_{2}{ }^{\prime}$, and $R_{\text {cre }}{ }^{\prime}$ in Equations 19 to 21 of different UE types due to the dynamic changing features of different time resource partitions with eICIC.

$$
\begin{gathered}
R_{1}{ }^{\prime}=\frac{\lambda_{1}}{A_{1}} \int_{0}^{\infty} \frac{1}{C_{1}+C(t)} d t \\
R_{2}{ }^{\prime}=(1-\beta) \frac{\lambda_{2}}{A_{2}} \int_{0}^{\infty} \frac{1}{C_{2}+C(t)} d t+\beta \frac{\lambda_{2}}{A_{2}} \int_{0}^{\infty} \frac{1}{C_{2}+C(t)^{\prime}} d t \\
R_{\text {cre }}{ }^{\prime}=\frac{\lambda_{2}}{A_{\text {cre }}} \int_{0}^{\infty} \frac{1}{C_{\text {cre } 1}+C(t)^{\prime}}-\frac{1}{C_{\text {cre } 2+C(t)^{\prime}}} d t
\end{gathered}
$$

Thus, the proposed CRE bias scheme can realize the load balance among different cells and increase the capacity in multi-tier heterogeneous cellular networks with appropriate CRE bias value configuration. However, $\mathrm{PUE}_{\text {cre }}$ will suffer the strong interference from MBS in the vicinity. Furthermore, in order to minimize the inter-cell interference, the eICIC technology using ABS ratio configuration is applied to utilize both the temporal and spatial separations among MUEs and PUEs. But the system capacity of multi-tier heterogeneous cellular networks is affected in terms of the inefficient resource utilization. Thus, how to improve the capacity by considering the tradeoff between CRE bias $B$ and ABS ratio $\beta$ is a big challenge, which has not been solved yet. In summary, a novel eICIC technology by jointly utilizing CRE bias and ABS ratio scheme is proposed by considering the fairness aspect among different users in Section 3 to minimize the inter-cell interferences among multi-tier heterogeneous cellular networks, improving the network throughput and QoS.

\section{Optimal CRE bias and ABS ratio solution based on fairness}

In this section, an optimal $\mathrm{CRE}$ bias and $\mathrm{ABS}$ ratio scheme is proposed by considering the fairness aspect among different UEs in multi-tier heterogeneous cellular networks. CRE technology can offload part of MUEs into small cells, and the data rate of a single MUE will improve with the increase of CRE bias value $B$. Thus, the proposed CRE bias scheme can realize the load balance and increase the capacity in multi-tier heterogeneous cellular networks with an appropriate CRE bias value configuration. However, $\mathrm{PUE}_{\text {cre }}$ will suffer the strong inter-cell interference from MBS. To minimize the interference, ABS ratio configuration is applied as an eICIC scheme to utilize both the temporal and spatial separations among MUEs and PUEs with the capacity deteriorated. Thus, in order to improve the capacity in terms of the tradeoff between CRE bias $B$ and ABS ratio $\beta$ is considered as an effective solution to minimize the interference among cells, which is critical for network capacity enhancement in multi-tier heterogeneous cellular networks.

Thus, an optimal CRE bias and ABS ratio solution is proposed in this paper by taking into account the fairness aspect of three types of UEs at different locations within the macrocell. The single UE's average ergodic rate $\bar{R}_{k}$ of the $k$ th type is denoted in Equation 22, where $N_{k}$ is the UE's average number per cell in Equations 6 to 8. The joint CRE and eICIC scheme allocates the radio resources to different UEs. Moreover, the tradeoff between system throughput and fairness exists and achieved in this paper with numerous results. The proportional fairness utility $P F$ is denoted by Equation 23. And the average capacity per area unit is denoted by $C_{a}$ in Equation 24.

$$
\begin{aligned}
& \bar{R}_{k}=\rho_{k} \frac{R_{k}}{N_{k}} \\
& \mathrm{PF}=\sum_{k=1}^{K} A_{k} \log \left(\bar{R}_{k}\right) \\
&=\left[A_{1} \log \left(\frac{(1-\beta) R_{1} \mathrm{ICIC}}{N_{1}}\right)+A_{2} \log \left(\frac{R_{2 \mathrm{ICIC}}}{N_{2}}\right)\right. \\
&\left.\quad+A_{\mathrm{cre}} \log \left(\frac{\beta R_{\text {cre ICIC }}}{N_{\mathrm{cre}}}\right)\right] \\
&=A_{1} \log \left(\frac{\lambda_{1}^{2}(1-\beta)}{A_{1}^{2} \lambda_{u}} \int_{0}^{\infty} \frac{1}{C_{1}+C(t)} d t\right) \\
&+A_{2} \log \left(\frac{\lambda_{2}^{2}(1-\beta)}{A_{2}^{2} \lambda_{u}} \int_{0}^{\infty} \frac{1}{C_{2}+C(t)} d t+\frac{\lambda_{2}^{2} \beta}{A_{2}^{2} \lambda_{u}} \int_{0}^{\infty} \frac{1}{C_{2}+C(t)^{\prime}} d t\right) \\
&+A_{\mathrm{cre}} \log \left(\frac{\lambda_{2}^{2} \beta}{A_{\mathrm{cre}^{2} \lambda_{u}}} \int_{0}^{\infty} \frac{1}{C_{1}+C(t)^{\prime}}-\frac{1}{C_{2}+C(t)^{\prime}} d t\right)
\end{aligned}
$$




$$
\begin{aligned}
& C_{a}=\sum_{k=1}^{K} \rho_{k} A_{k} R_{k} W_{k} \\
& =W\left[\begin{array}{l}
(1-\beta) A_{1} R_{1_{\mathrm{ICIC}}}+A_{2} R_{\mathrm{ICIC}_{\mathrm{ICI}}}+\beta A_{\left.\mathrm{cre} R_{\text {cre }_{I C I C}}\right]} \\
=W\left[\begin{array}{l}
(1-\beta) \lambda_{1} \int_{0}^{\infty} \frac{1}{C_{1}+C(t)} d t+(1-\beta) \lambda_{2} \int_{0}^{\infty} \frac{1}{C_{2}+C(t)} d t \\
+\beta \lambda_{2} \int_{0}^{\infty} \frac{1}{C_{2}+C(t)^{\prime}} d t
\end{array}\right] \\
=W \lambda_{2} \int_{0}^{\infty} \frac{1}{C_{\mathrm{cre} 1+C(t)^{\prime}}}-\frac{1}{C_{\mathrm{cre} 2}+C(t)^{\prime}} d t
\end{array}\right] \\
& =W\left[\begin{array}{l}
(1-\beta) \int_{0}^{\infty} \frac{\lambda_{1}}{C_{1}+C(t)}+\frac{\lambda_{2}}{C_{2}+C(t)} d t+\beta \lambda_{2} \int_{0}^{\infty} \frac{1}{C_{1}+C(t)^{\prime}} d t
\end{array}\right]
\end{aligned}
$$

The proportional fairness utility was originally proposed by Kelly in the context of rate control of wired networks [22], maximizing the logarithmic utility function, $y=\sum_{i=1}^{U} \log \left(T_{i}\right)$, where $T_{i}$ is the throughput of user $i$ and $U$ is the total number of users in the system, yields a good balance between system throughput and fairness. Therefore, in order to guarantee the fairness of the three types of UEs in multi-tier heterogeneous cellular networks, the optimization model is defined as a multiobjective decision-making problem in Equation 25 with constraints of CRE bias $B$ and ABS ratio $\beta$, where $F(B, \beta)$ is the objective function, $\phi(B, \beta)$ is the constraint, and CRE bias $B$ and ABS ratio $\beta$ are variables.

$$
\begin{aligned}
& F(B, \beta)=\left\{\begin{array}{l}
\max P F(B, \beta) \\
\max C_{a}(B, \beta)
\end{array}\right. \\
& \text { s.t. } \phi(B, \beta)=\left\{\begin{array}{c}
0 \leq B \leq 2 \mathrm{~dB} \\
1 / 8 \leq \beta \leq 7 / 8
\end{array}\right.
\end{aligned}
$$

Generally, the multi-objective functions cannot reach the same maximum result in meanwhile. Therefore, to solve the multi-objective problem, the lexicographic method is applied which sorts the objective functions according to the importance. The optimal solution of $m$ th objective is based on the previous optimum solution of $(m-1)$ th objective. Thus, the multi-objective optimal solution for Equation 25 is achieved finally in Equation 26. In our solution, guaranteeing fairness is more important than improving capacity; thus, we choose maximizing PF as the first objective and maximizing the $C_{a}$ as the second objective. In addition, applying the lexicographic method can reduce almost half the amount of calculation and can realize guaranteeing fairness and improving capacity in meanwhile. The algorithm of optimal CRE bias and ABS ratio is as Algorithm 1.

$$
\begin{aligned}
& f_{1}\left(\{B, \beta\}^{(1)}\right)=\max _{\{B, \beta\} \in R_{0}} \operatorname{PF}(B, \beta) \\
& f_{2}\left(\{B, \beta\}^{(2)}\right)=\max _{\{B, \beta\} \in R_{1}} C_{a}(B, \beta) \\
& R_{1}=\left\{\{B, \beta\} \mid\{B, \beta\} \in R_{0}\right\} \\
& R_{0}=\{\{B, \beta\}=\{[0 \mathrm{~dB}, 20 \mathrm{~dB}],[1 / 8,7 / 8]\}\}
\end{aligned}
$$

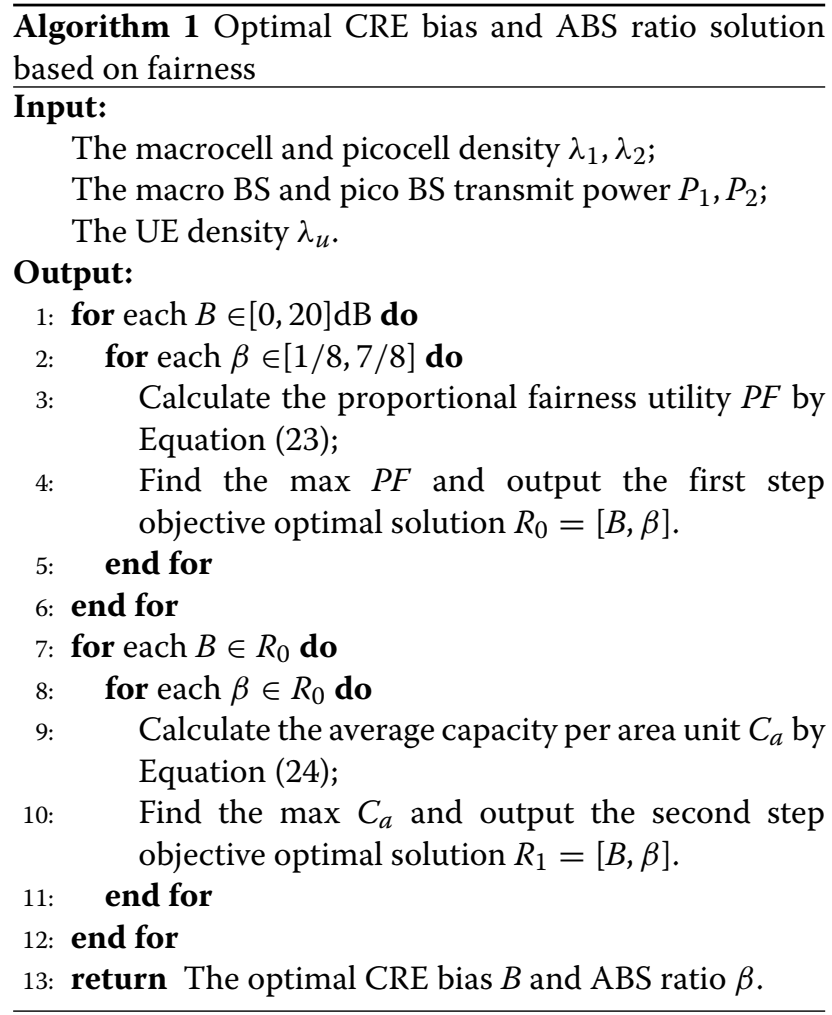

\section{Results and analysis}

In this section, numerical simulation is performed with different scenarios and results are analyzed thoroughly. Macrocells and picocells are deployed with densities of $\lambda_{1}=4.62$ and $\lambda_{2}=K_{P} \lambda_{1} \mathrm{BS} / \mathrm{km}^{2}$, respectively. And the transmit power for macrocell and picocell is $P_{1}=46$ and $P_{2}=30 \mathrm{dBm}$. The system bandwidth is defined by $W=10 \mathrm{MHz}$, and the path loss exponent is denoted by $\alpha=4$.

\subsection{Capacity analysis of stand-alone effects by BS density, CRE, and eICIC}

Considering different parameters that affect the performance of system capacity in multi-tier heterogeneous cellular networks, key parameters, such as the BS density, CRE, and eICIC, are discussed with numerical results in terms of different ratios of PUE, system throughput of various cells.

In terms of the effects of BS density and CRE bias values to the system capacity, results are discussed and analyzed thoroughly below. First, the traffic offloading from macrocells to picocells is depicted in Figure 3. The PUE's ratio increases with the surge of CRE bias value when the picocell's density is constant. With the increase of picocell's density, PUE's ratio will also increase. By using the CRE technology, the over-loaded macrocell can offload part of its traffic to picocells which can realize a load balance in multi-tier heterogeneous cellular networks. 


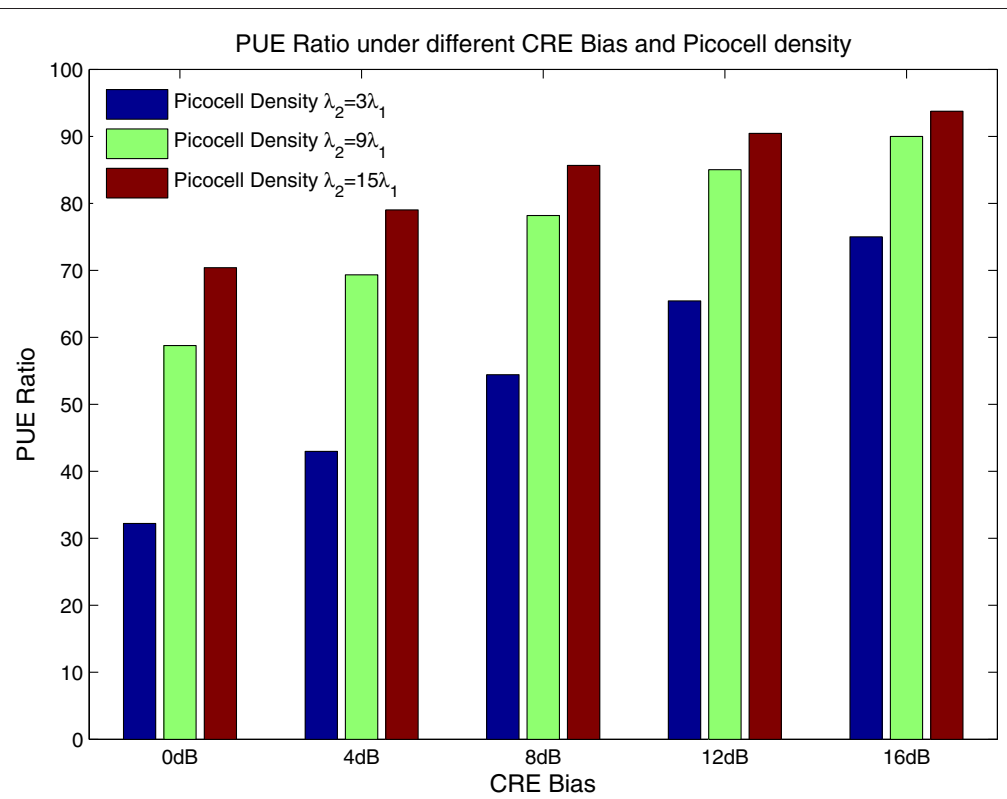

Figure 3 PUE's ratio under different CRE bias and picocell's density.

Besides, Figure 4 depicts the effect of CRE bias and picocell's density on the average capacity per cell under different CRE bias values. As the CRE bias increases, more MUEs with a low SINR value are associated with picocells and the PUE $\mathrm{Pre}_{\mathrm{c}} \mathrm{s}$ are associated to BSs that are not offering the strongest received signal to minimize the inter-cell interference. Therefore, with the increase of CRE bias, the throughput of macrocells increases and the average throughput of picocells decreases.

In terms of the effect of utilizing the eICIC technology to the system capacity, Figure 5 denotes the effect of $A B S$ ratio value in eICIC technology and picocell's density on the average throughput per cell. As the ABS ratio increases, more MUEs are mute on specific subframes, which provide more $\mathrm{PUE}_{\text {cre }} \mathrm{S}$ with more time resources. Therefore, with the increase of ABS ratio, the throughput of a macrocell decreases and the throughput of a picocell increases owning to the efficient time-domain resource configuration among macrocells and picocells using ABS ratio.

\subsection{Optimal CRE bias and ABS ratio technology}

The average capacity per area is depicted in Figure 6 in multi-tier heterogeneous networks, which increases as

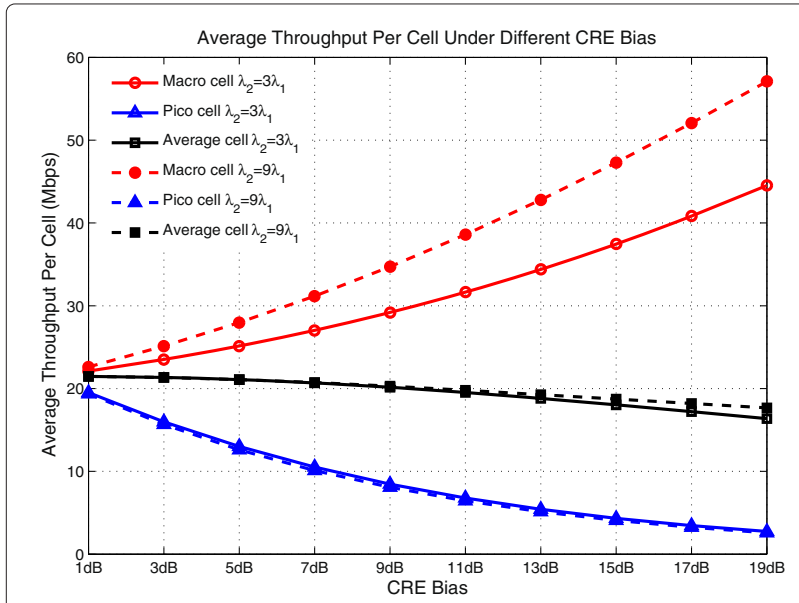

Figure 4 The average capacity per cell under different CRE bias.

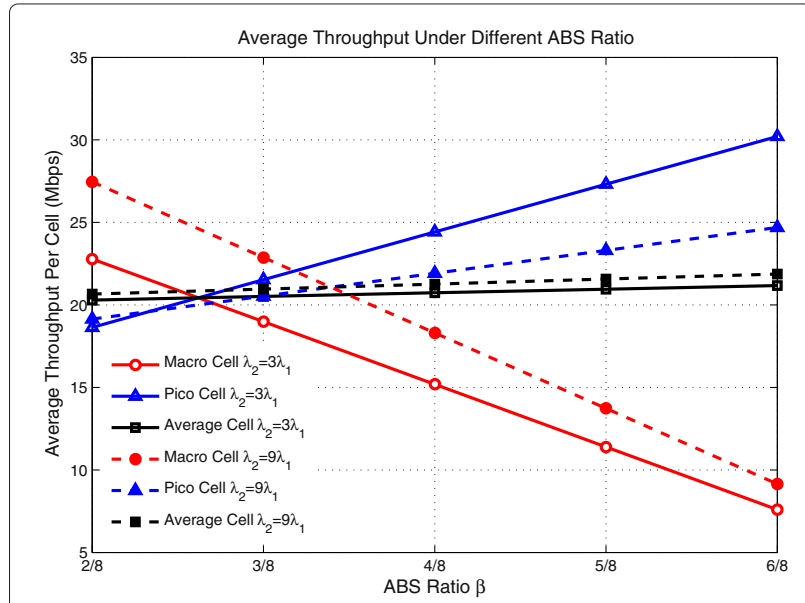

Figure 5 The average capacity per cell under different ABS ratio. 


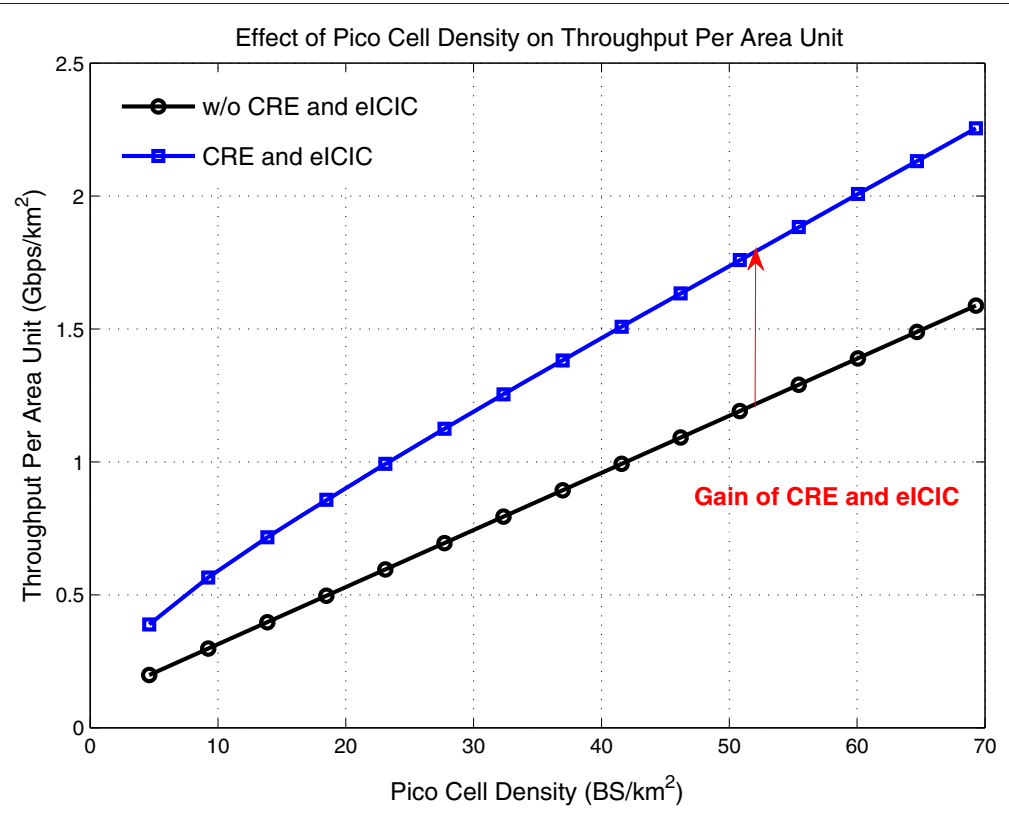

Figure 6 Effect of picocell's density to average capacity per area unit with/without CRE and eICIC.

the picocell's density increases. The joint CRE and eICIC scheme can improve the network capacity. In order to provide the service with a requirement of $1.3 \mathrm{Gbps} / \mathrm{km}^{2}$ and $10-\mathrm{MHz}$ system bandwidth, the picocell's density is about $55 \mathrm{BS} / \mathrm{km}^{2}$ (about 12 times higher than macrocell's density) without using CRE and eICIC. Importantly, by using the CRE and eICIC scheme, the picocell's density decreases significantly to $30 \mathrm{BS} / \mathrm{km}^{2}$ (about 6 times higher than macrocell's density) which can minimize the inter-cell interference among macrocells and densely deployed picocells. In addition, under the same picocell's density, the joint CRE and eICIC technology can improve the network capacity over $40 \%$.

Furthermore, the fairness effect is considered by PF utility value, which is a function of both CRE bias and ABS ratio values as depicted in Figure 7. The PF utility can achieve the optimal value with the increase of CRE bias and ABS ratio values. Moreover, the average

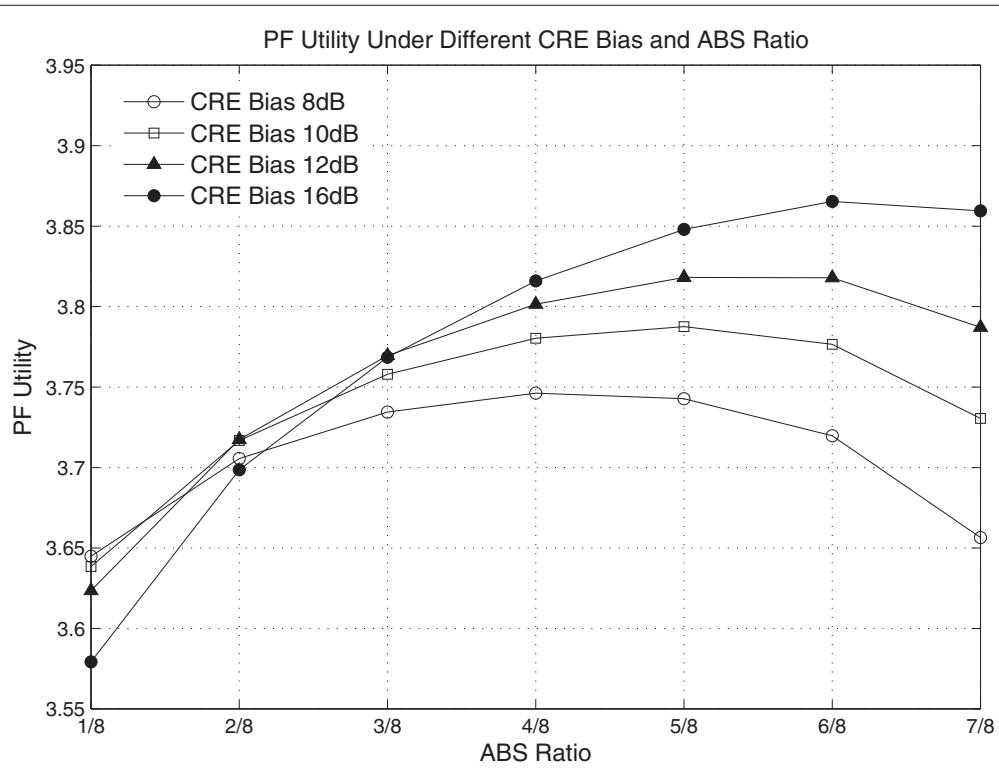

Figure 7 PF utility with different CRE bias and ABS ratio. 


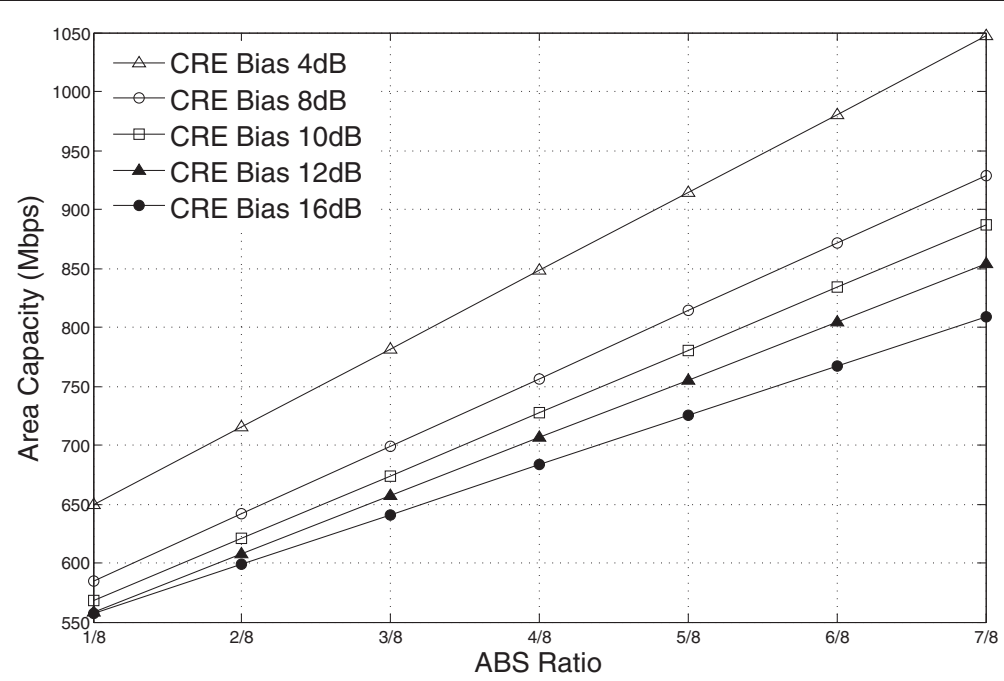

Figure 8 Average capacity with different CRE bias and ABS ratio.

capacity increases with the increase of ABS ratio values in Figure 8, which depicts that the capacity of picocells will increase by using the ABS ratio scheme. And the average capacity decreases as CRE bias becomes larger.

By utilizing the lexicographic method, the optimal CRE bias and ABS ratio are achieved in multi-tier heterogeneous cellular networks. In the case of picocell's density $\lambda_{2}=6 \lambda_{2}$, the PF utility has little difference when the CRE bias is from 8 to $12 \mathrm{~dB}$ and $\mathrm{ABS}$ ratio is from $3 / 8$ to $6 / 8$ as shown in Figure 7. In order to maximum the average capacity per cell, the optimal CRE bias is $12 \mathrm{~dB}$ and ABS ratio is $6 / 8$. Similarly, when the picocell's density $\lambda_{2}=$
$12 \lambda_{2}$, the optimal CRE bias is $10 \mathrm{~dB}$ and ABS ratio is $5 / 8$. In addition, the optimal CRE bias and $A B S$ ratio are less affected by picocell's density. Therefore, simulation results show that when the CRE bias is from 8 to $12 \mathrm{~dB}$ and $A B S$ ratio is from $4 / 8$ to $6 / 8$, the optimal solution is achieved which can improve the capacity by $40 \%$ in terms of the fairness among different UEs in multi-tier heterogeneous cellular networks.

\subsection{LTE-A system-level simulation results of novel elCIC technology}

A network layout with co-channel deployment of macrocell and picocell as defined in [7] is simulated. The

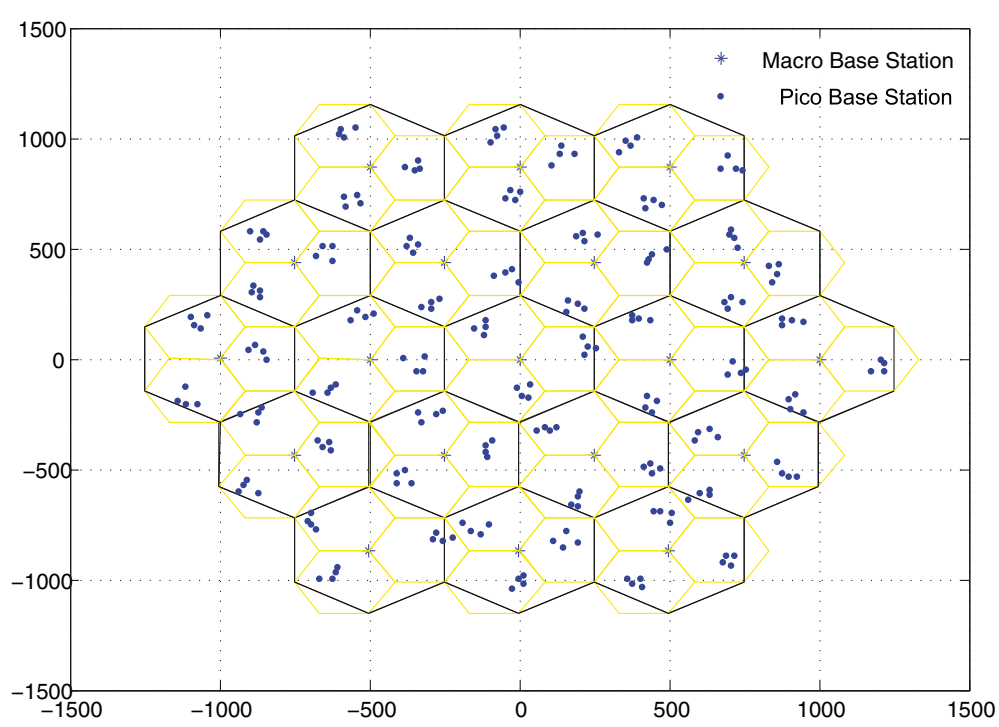

Figure 9 The network topology of two-tier HetNet. 
Table 1 Simulation parameters

\begin{tabular}{lcc}
\hline & Macrocell & Picocell \\
\hline Cellular layout & 19 cell sites, 3 sectors per site & 4 picocells per sector \\
Number of UEs & 60 per macro cell & 60 per macro cell \\
Distance-dependent path loss & ITU urban macro(UMa) [7] & ITU urban micro(UMi) [7] \\
Shadowing standard deviation & $8 \mathrm{~dB}$ & $10 \mathrm{~dB}$ \\
Penetration loss & $0 \mathrm{~dB}$ & $0 \mathrm{~dB}$ \\
Total eNodeB Tx power & $46 \mathrm{dBm}$ & $30 \mathrm{dBm}$ \\
Antenna configuration & $2 \times 2$ (uncorrelated) & 2 (uncorrelated) \\
Antenna pattern & $3 \mathrm{D}$ & $2 \mathrm{D}$ \\
Antenna height & $25 \mathrm{~m}$ & $10 \mathrm{~m}$ \\
Antenna gain & $14 \mathrm{dBi}$ & $5 \mathrm{dBi}$ \\
Carrier frequency & $2 \mathrm{GHz}$ & $2 \mathrm{GHz}$ \\
System bandwidth & $10 \mathrm{MHz}$ & $10 \mathrm{MHz}$ \\
Traffic model & Full buffer, full load & Full buffer, full load \\
\hline
\end{tabular}

network topology consists of the standard three-sector macro BSs, complemented with a set of 4 pico BSs per sector as in Figure 9. For scenarios with CRE and eICIC enabled technologies, we assume that CRE bias $10 \mathrm{~dB}$ and $A B S$ ratio $5 / 8$ are suitable for all pico BSs. UEs are assumed to be LTE Release 10 compliant, so they can support separate reporting of CSI for ABS and non-ABS subframes. The default simulation parameters are summarized in Table 1.

Figure 10 shows the cumulative distribution function (CDF) of PUE's SINR. By using the eICIC technology, the SINR of PUEs can be promoted because PUEs are scheduled on ABSs without the interference from macrocells with the appropriate ABS configuration.
Figure 11 shows the CDF of throughput of different UEs. By using the CRE and eICIC technology, the throughput of PUE can be promoted and the average throughput will also increase. However, the throughput of MUE will decrease because of limited time resource. Furthermore, the throughput gap between MUE and PUE is decreased and more fair than scenario without CRE and eICIC. In a word, the joint using of CRE and eICIC can improve throughput and realize fairness meanwhile.

\section{Conclusion}

In this paper, the joint CRE and eICIC scheme is proposed to minimize the inter-cell interference in multi-tier

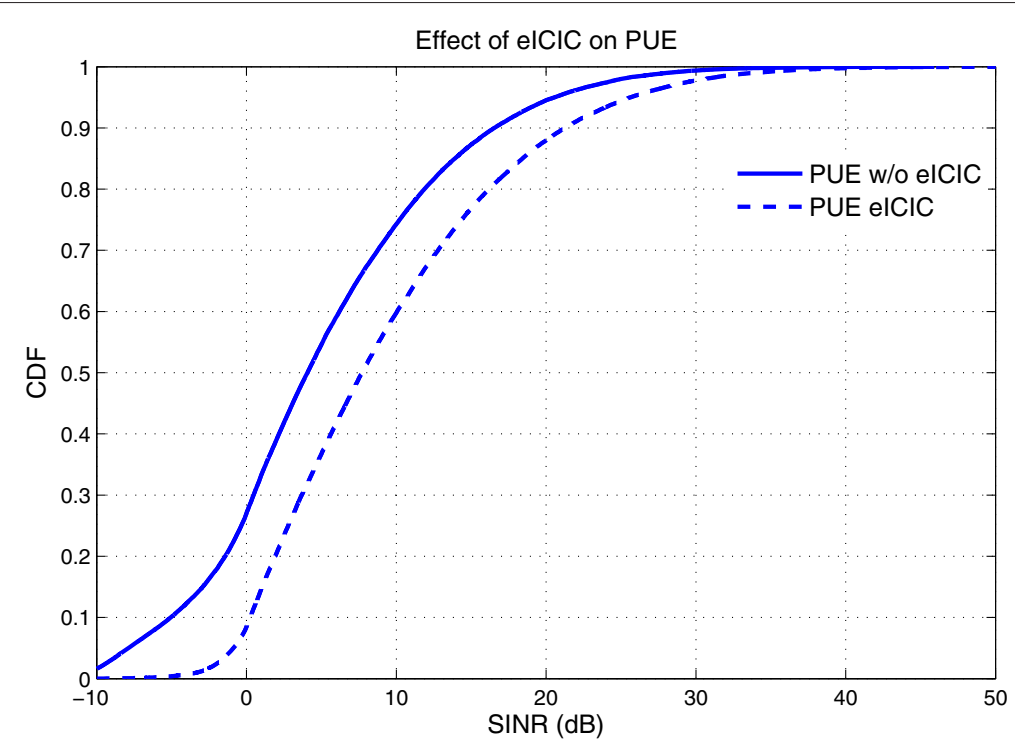

Figure 10 Cumulative distribution function of PUE's SINR. 


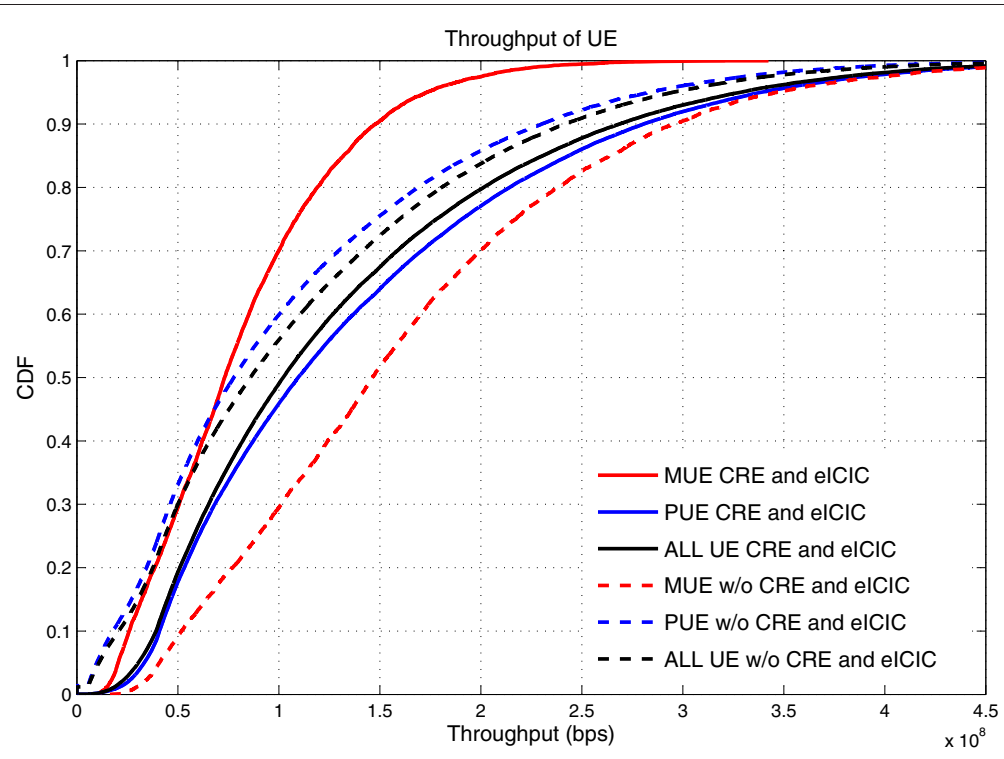

Figure 11 Cumulative distribution function of throughput for different UEs.

heterogeneous cellular networks. And the optimal CRE bias and ABS ratio solutions are achieved by taking into account the fairness effect among different UEs. The multi-objective decision-making scheme is designed to maximize the PF utility and the aera capacity. Simulation results show that when the CRE bias is from 8 to $12 \mathrm{~dB}$ and $\mathrm{ABS}$ ratio is from $4 / 8$ to $6 / 8$, the optimal solution is achieved which can improve the capacity by $40 \%$, which considers about the fairness among different UEs.

\section{Competing interests}

The authors declare that they have no competing interests.

\section{Acknowledgements}

This work was sponsored by the National Natural Science Foundation of China (61201152, 61227801), the National High-tech R\&D Program (863 Program 2014AA01A707)

Received: 1 November 2014 Accepted: 16 February 2015

Published online: 10 March 2015

\section{References}

1. P Mogensen, K Pajukoski, B Raaf, E Tiirola, L Eva, I Kovacs, G Berardinelli, LGU Garcia, L Hu, AF Cattoni, in IEEE 2012 Globecom Workshops. B4G local area: high level requirements and system design (Anaheim, USA, 2012), pp. 613-617

2. N Bhushan, Junyi, L, D Malladi, R Gilmore, Network densification: the dominant theme for wireless evolution into 5G. IEEE Commun. Mag. 52(2), 82-89 (2014)

3. MAM Al-Shibly, MH Habaebi, J Chebil, in IEEE Control Syst. Graduate Res. Colloquium. Carrier aggregation in long term evolution-advanced (Shah Alam, Selangor, 2012), pp. 154-159

4. A Davydov, G Morozov, I Bolotin, A Papathanassiou, in IEEE 2013 Globecom Workshops. Evaluation of joint transmission CoMP in C-RAN based LTE-A HetNets with large coordination areas (Atlanta, USA, 2013), pp. 801-806

5. S Parkvall, E Dahlman, G Jongren, S Landstrom, L Lindbom, Heterogenous network deployments in LTE. Ericsson Rev. 2, 34-38 (2011)
6. K Hiltunen, in IEEE Vehicular Technology Conference. Comparison of different network densification alternatives from the LTE downlink (San Francisco, USA, 2011), pp. 1-5

7. 3GPP TR 36.814 (V9.0.0), Evolved universal terrestrial radio access (E-UTRA); further advancements for E-UTRA physical layer aspects (Release 9), Mar. 2010

8. J Xu, J Wang, Y Zhu, Y Yang, X Zheng, S Wang, L Liu, K Horneman, Y Teng, Cooperative distributed optimization for the hyper-dense small cell deployment. IEEE Commun. Mag. 52(5), 61-67 (2014)

9. H Insoo, S Bongyong, SS Soliman, A holistic view on hyper-dense heterogeneous and small cell networks. IEEE Commun. Mag. 51(6), 20-27 (2013)

10. 3GPP R1-083813, Range expansion for efficient support of heterogeneous networks. (Qualcomm, Europe, Prague, Czech Republic, 2008)

11. 3GPP R1-104661, Comparison of Time-Domain elCIC Solutions. (LG Electronics, Madrid, Spain, 2010)

12. K Okino, T Nakayama, C Yamazaki, H Sato, Y Kusano, in IEEE 2011 ICC Workshops. Pico cell range expansion with interference mitigation toward LTE-advanced heterogeneous networks (Kyoto, Japan, 2011), pp. 1-5

13. M Vajapeyam, A Damnjanvic, J Montojo, T Ji, Y Wei, D Malladi, in IEEE 2011 ICC Workshops. Downlink FTP performance of heterogeneous networks for LTE-Advanced (Kyoto, Japan, 2011), pp. 1-5

14. R Madan, J Borran, A Sampath, N Bhushan, A Khandekar, T Ji, Cell association and interference coordination in heterogeneous LTE-A cellular networks. IEEE JSAC. 28(9), 1479-1489 (2010)

15. M Shirakabe, A Morimoto, N Miki, in IEEE International Symposium on Wireless Communication Systems. Performance evaluation in heterogeneous networks employing time-domain inter-cell interference coordination and cell range expansion for LTE-advanced downlink (Aachen, Germany, 2011), pp. 844-848

16. H-S Jo, Y-J Sang, P Xia, JG Andrews, Heterogeneous cellular networks with flexible cell association: a comprehensive downlink SINR analysis. IEEE Trans. Wireless Commun. 11(10), 3484-3495 (2012)

17. TD Novlan, RK Ganti, A Ghosh, JG Andrews, Analytical evaluation of fractional frequency reuse for heterogeneous cellular networks. IEEE Trans. Commun. 60(7), 2029-2039 (2012)

18. M Cierny, H Wang, R Wichman, Z Ding, C Wijting, On number of almost blank subframes in heterogeneous cellular networks. IEEE Trans. Commun. 12(10), 5061-5073 (2013)

19. S Mukherjee, I Guvenc, in Proc 2011 Asilomar Conf. Signals, Syst. Comput. Effects of range expansion and interference coordination on capacity and fairness in heterogeneous networks (Pacific Grove, USA, 2011), pp. 1855-1859 
20. S Singh, JG Andrews, Joint resource partitioning and offloading in heterogeneous cellular networks. IEEE Trans. Wireless Commun. 13(2), 888-901 (2014)

21. SD Wang, J Wang, J Xu, Y Teng, K Horneman, Fairness guaranteed cooperative resource allocation in femtocell networks. Wireless Pers. Commun. 72, 957-973 (2013)

22. FP Kelly, Charging and rate control for elastic traffic. Euro. Trans. Telecommun. 8, 7-20 (1997)

Submit your manuscript to a SpringerOpen ${ }^{\circ}$ journal and benefit from:

- Convenient online submission

- Rigorous peer review

- Immediate publication on acceptance

- Open access: articles freely available online

- High visibility within the field

- Retaining the copyright to your article

Submit your next manuscript at $\gg$ springeropen.com 